\title{
Bing-Neel syndrome
}

\section{Síndrome de Bing-Neel}

Artemios K. Artemiadis ${ }^{1,2}$, Aspasia Terentiou², Dimosthenis Kolokythopoulos ${ }^{3}$, Nikos Triantafyllou ${ }^{1}$, George Nikolaou ${ }^{2}$

A 75-year-old man with IgM-kappa restricted Waldenström macroglobulinemia (WM) was admitted due to paraparesis and sensory deficits of the lower extremities. Cerebrospinal fluid analysis revealed 140 cells $/ \mathrm{mm}^{3}$, increased protein (295mg\%), albumin quotient (16.57) and the presence of IgM-kappa chains in immunofixation electrophoresis. Cytology showed lymphoplasmacytoid cells. Magnetic resonance imaging (MRI) demonstrated spinal cord hydromelia and gadolinium-enhancing root lesions (Figure). These findings suggested central nervous system (CNS) involvement of WM or type A Bing-Neel syndrome ${ }^{1}$. In Bing-Neel syndrome, MRI usually discloses tumoral or diffuse CNS abnormalities with gadolinium-enhancing leptomeningeal, parenchymal or cranial nerve lesions ${ }^{2}$. Chemotherapy (intrathecal methotrexate, rituximab, cyclophosphamide and vincristine) was dispensed with marked clinical improvement.
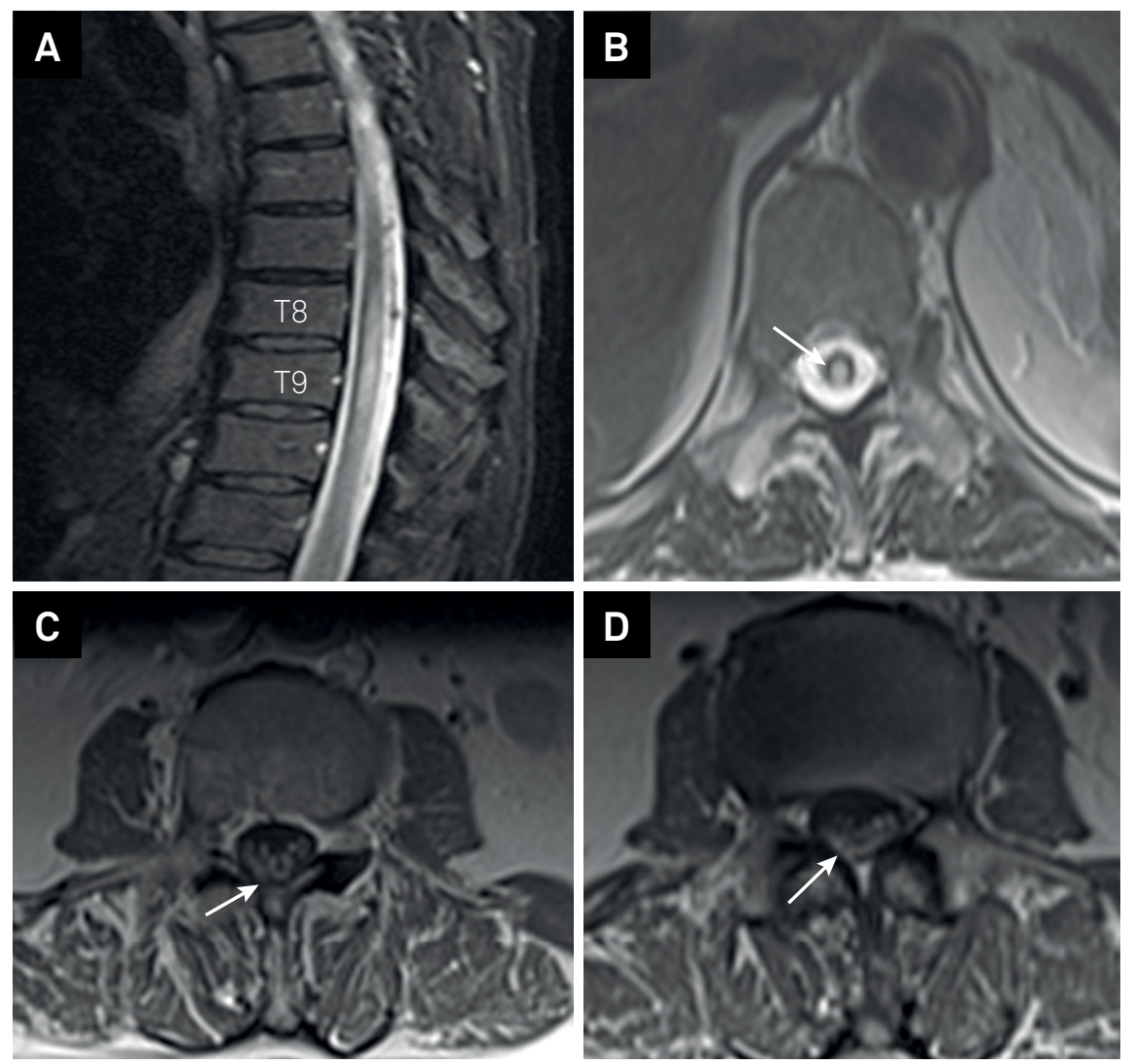

Figure. Sagittal (A) and axial (B) T2-weighted MRI show marked hydromyelia at T8-T9 level. Axial lumbar T1-weighted with gadolinium MRIs (C,D) show diffuse enhancement of cauda equina's nerve roots (arrows).

\section{References}

1. Fintelmann F, Forghani R, Schaefer PW, Hochberg EP, Hochberg FH. Bing-Neel Syndrome revisited.

Clin Lymphoma Myeloma. 2009;9(1):104-6.

https://doi.org/10.3816/CLM.2009.n.028
2. Malkani RG, Tallman M, Gottardi-Littell N, Karpus W, Marszalek L, Variakojis D et al. Bing-Neel syndrome: an illustrative case and a comprehensive review of the published literature. J Neurooncol. 2010;96(3):301-12. https://doi.org/10.1007/s11060-009-9968-3

\footnotetext{
${ }^{1}$ Kapodistrian University of Athens, School of Medicine, Aeginition Hospital, 1st Department of Neurology, Athens, Greece;

${ }^{2}$ NIMTS Hospital, Department of Neurology, Athens, Greece;

${ }^{3}$ NIMTS Hospital, Department of Hematology, Athens, Greece.

Correspondence: Artemiadis K. Artemios; Monis Petraki Str., 10-12, GR-115-21, Athens, Greece; Email: kmwartem@yahoo.com

Conflict of interest: There is no conflict of interest to declare.

Received 18 August 2016; Accepted 21 November 2016.
} 


\section{Erratum}

Bing-Neel syndrome.

Arq Neuropsiquiatr 2017;75(4):262. https://doi.org/10.1590/0004-282X20170017

The name of the authors:

Artemiadis K Artemios ${ }^{1,2}$, Terentiou Aspasia², Kolokythopoulos Dimosthenis ${ }^{3}$, Triantafyllou Nikos ${ }^{1}$, Nikolaou George ${ }^{2}$

Should be:

Artemios K. Artemiadis ${ }^{1,2}$, Aspasia Terentiou ${ }^{2}$, Dimosthenis Kolokythopoulos ${ }^{3}$, Nikos Triantafyllou ${ }^{1}$, George Nikolaou ${ }^{2}$ 